\title{
Identification et quantification de 81 résidus médicamenteux au sein d'une zone de rejet végétalisée : rétention différenciée des compartiments eau-sol-plantes \\ Identification and quantification of 81 pharmaceutical compounds in a surface flow treatment wetland: Differentiated retention in water-soil-plants
}

\author{
Maximilien Nuel, Julien Laurent, Paul Bois, Dimitri Heintz et Adrien Wanko
}

Volume 30, numéro 1, 2017

Reçu le 5 octobre 2016, accepté le 14 mars 2017

URI : https://id.erudit.org/iderudit/1040063ar

DOI : https://doi.org/10.7202/1040063ar

Aller au sommaire du numéro

Éditeur(s)

Université du Québec - INRS-Eau, Terre et Environnement (INRS-ETE)

ISSN

1718-8598 (numérique)

Découvrir la revue

Citer cet article

Nuel, M., Laurent, J., Bois, P., Heintz, D. \& Wanko, A. (2017). Identification et quantification de 81 résidus médicamenteux au sein d'une zone de rejet végétalisée : rétention différenciée des compartiments eau-sol-plantes. Revue des sciences de l'eau / Journal of Water Science, 30(1), 49-55.

https://doi.org/10.7202/1040063ar

\section{Résumé de l'article}

La présence de résidus médicamenteux dans les cours d'eau est notamment due à une épuration incomplète de ces derniers dans les stations de traitement des eaux usées (STEU). Leur impact potentiel sur la faune aquatique a été mis en lumière par la féminisation des poissons causée par la présence d'hormones à quelques $n g \cdot \mathrm{L}^{-1}$ dans l'eau. Cette étude fait un focus de plusieurs mois sur les performances d'épuration d'un filtre planté de roseaux à deux étages et écoulement vertical (FPRvv), ainsi que de la zone de rejet végétalisée (ZRV) située en aval du FPRvv. La STEU est dimensionnée pour 808 équivalents habitants et se situe dans le Haut-Rhin, France. La ZRV est de type mare et reçoit les eaux en sortie de FPRvv et les restitue au milieu récepteur; 81 résidus pharmaceutiques y sont recherchés dans les échantillons d'eau, de plantes et de sol. Les résultats mettent en évidence que le nombre de micropolluants a augmenté en période hivernale. Une concentration maximum fut atteinte en novembre 2016 pour l'amitriptyline (antidépresseur) à $2,9 \mu \mathrm{g} \cdot \mathrm{L}^{-1}$ alors qu'en été ce fut l'alpha éthinylestradiol (contraceptif hormonal) à $54 \mu \mathrm{g} \cdot \mathrm{L}^{-}{ }^{1}$. Le FPRvv a abattu majoritairement entre 30 et $70 \%$ des micropolluants. La ZRV a quant à elle complété cette épuration en abattant là aussi majoritairement entre 30 et $70 \%$ de la pollution en sortie de FPRvv (soit une participation à l'épuration globale comprise entre 30 et $70 \%$ ). L'étude des plantes et du sol de la ZRV a mis en lumière que ces matrices captaient des micropolluants et les cumulaient dans le temps. Les concentrations sont de l'ordre du pg. $\mathrm{g}^{-1}$ de matière sèche (plante) ou de boue égouttée. La détection des molécules diffère qualitativement et quantitativement d'une plante à l'autre, laissant supposer un transfert spécifique d'une molécule à une espèce. 


\section{IDENTIFICATION ET QUANTIFICATION DE 81 RÉSIDUS MÉDICAMENTEUX AU SEIN D'UNE ZONE DE REJET VÉGÉTALISÉE : RÉTENTION DIFFÉRENCIÉE DES COMPARTIMENTS EAU-SOL-PLANTES}

Identification and quantification of 81 pharmaceutical compounds in a surface flow treatment wetland: Differentiated retention in water-soil-plants

$$
\text { MAXIMILIEN NUEL }{ }^{1,2 *} \text { JULIEN LAURENT', PAUL BOIS', DIMITRI HEINTZ², ADRIEN WANKO' }
$$

${ }^{1}$ Université de Strasbourg, Institut National des Sciences Appliquées, Département mécanique des fluides et rhéologie, Icube Laboratoire des sciences de l'ingénieur, de l'informatique et de l'imagerie, 2, rue Boussingault, 67000 Strasbourg, France ${ }^{2}$ Institut de Biologie Moléculaire de Plantes, CNRS, Plateforme métabolomique, UPR 2357, 12, rue du Général Zimmer, F-67084 Strasbourg, France

Reçu le 5 octobre 2016, accepté le 14 mars 2017

\section{RÉSUMÉ}

La présence de résidus médicamenteux dans les cours d'eau est notamment due à une épuration incomplète de ces derniers dans les stations de traitement des eaux usées (STEU). Leur impact potentiel sur la faune aquatique a été mis en lumière par la féminisation des poissons causée par la présence d'hormones à quelques ng. $\mathrm{L}^{-1}$ dans l'eau. Cette étude fait un focus de plusieurs mois sur les performances d'épuration d'un filtre planté de roseaux à deux étages et écoulement vertical (FPRvv), ainsi que de la zone de rejet végétalisée (ZRV) située en aval du FPRvv. La STEU est dimensionnée pour 808 équivalents habitants et se situe dans le HautRhin, France. La ZRV est de type mare et reçoit les eaux en sortie de FPRvv et les restitue au milieu récepteur; 81 résidus pharmaceutiques y sont recherchés dans les échantillons d'eau, de plantes et de sol. Les résultats mettent en évidence que le nombre de micropolluants a augmenté en période hivernale.
Une concentration maximum fut atteinte en novembre 2016 pour l'amitriptyline (antidépresseur) à $2,9 \mu \mathrm{g} \cdot \mathrm{L}^{-1}$ alors qu'en été ce fut l'alpha éthinylestradiol (contraceptif hormonal) à $54 \mu \mathrm{g} \cdot \mathrm{L}^{-1}$. Le FPRvv a abattu majoritairement entre 30 et $70 \%$ des micropolluants. La ZRV a quant à elle complété cette épuration en abattant là aussi majoritairement entre 30 et $70 \%$ de la pollution en sortie de FPRvv (soit une participation à l'épuration globale comprise entre 30 et $70 \%$ ). Létude des plantes et du sol de la ZRV a mis en lumière que ces matrices captaient des micropolluants et les cumulaient dans le temps. Les concentrations sont de l'ordre du pg.g ${ }^{-1}$ de matière sèche (plante) ou de boue égouttée. La détection des molécules diffère qualitativement et quantitativement d'une plante à l'autre, laissant supposer un transfert spécifique d'une molécule à une espèce.

Mots-clés : Zone de rejet végétalisée, filtre planté de roseaux, médicaments, rendement d'épuration, micropolluant, saisonnalité. 


\section{ABSTRACT}

The occurrence of pharmaceutical compounds (PHc) in the aquatic environment is due to the incomplete micropollutant removal efficiencies of Wastewater Treatment Plants (WWTPs). As a consequence, some $\mathrm{PHc}$ in rivers are known to be responsible for the feminization of male fishes resulting from exposure to waterborne hormones, even at concentrations of a few $n g \cdot \mathrm{L}^{-1}$. This study involved the monitoring of $\mathrm{PHc}$ removal efficiencies in a WWTP located in Haut-Rhin, France, over the course of one year. The WWTP is composed of vertical flow constructed wetlands (VFCWs) followed by a surface flow treatment wetland (SFTW). The VFCW is sized to treat 808 people equivalents. Water, plants and soil from the VFCW and SFTW were sampled every two months and 81 PHc were investigated. Initial results show an increase in the number of PHc during winter campaigns, with maximum concentrations reached in November 2016 by amitriptyline (antidepressant) at $2.9 \mu \mathrm{g} \cdot \mathrm{L}^{-1}$. During the summer, ethinylestradiol (hormone) was the highest $\mathrm{PHc}$ concentration detected at $54 \mu \mathrm{g} \cdot \mathrm{L}^{-1}$ in wastewater. VFCW removal efficiencies of $\mathrm{PHc}$ ranged from $30 \%$ to $70 \%$ and were completed by SFTW removal efficiencies (30-70\%). Concerning plant and soil samples from the SFTW, PHc concentrations were detected at $\mathrm{pg} \cdot \mathrm{g}^{-1}$ levels $\left(10^{-12} \mathrm{~g} \cdot \mathrm{g}^{-1}\right.$ of dried plants or sludge $)$. These results illustrate the PHc cumulative abilities of plants and soil. Moreover, the presence and concentrations of PHc compounds depend on plant type, which suggests a specific relationship between $\mathrm{PHc}$ compounds and plant species..

\section{Key Words: Micropollutant, pharmaceutical compounds, removal efficiency, seasonality, surface flow treatment wetland, vertical flow constructed wetland.}

\section{INTRODUCTION}

À la fin des années 1990, les composés pharmaceutiques ont rapidement attiré l'attention du fait de leur implication entre autres dans la féminisation des poissons dans les cours d'eau (PURDOM et al., 1994; SUMPTER et JOBLING 1995; LARSSON et al., 1999). Même à l'état de traces dans les eaux, ces composés peuvent avoir des conséquences importantes sur l'écotoxicologie locale (LARSSON et al., 1999). Parallèlement à ce sujet, les collectivités (seules ou associées à d'autres) se sont dotées de stations d'épuration d'eaux usées (STEU) pour la dépollution des eaux usées domestiques avant leur rejet dans le milieu récepteur. Depuis une dizaine d'années, de nouveaux types d'ouvrages sont apparus à l'interface entre les STEU et les milieux superficiels : les zones de rejet végétalisées (ZRV). Ces dernières sont issues du génie écologique et tendent à recréer une zone humide en utilisant la place disponible sur le site d'implantation de la STEU (BOUTIN et PROST-BOUCLE, 2012). Ces ouvrages reçoivent tout ou partie des eaux de sortie de STEU alors qu'elles sont encore chargées en micropolluant à des concentrations pouvant aller jusqu'au $\mu \mathrm{g} \cdot \mathrm{L}^{-1}$ (LI, 2014). Les ZRV ont montré des capacités de potentialités de rétention, de dégradation et de dissipation de ces polluants (ZHANG et al., 2014). Ce travail a pour objectif d'identifier et de quantifier les résidus médicamenteux dans les ZRV ainsi que leur dispersion vers les plantes et boues décantées.

\section{MATÉRIELS ET MÉTHODES}

\subsection{Site d'étude}

Le site d'étude est une STEU de $808 \mathrm{EH}$ recevant les eaux usées domestiques ainsi qu'une partie des eaux de voirie et de ruissellement des communes de Lutter et de Raedersdorf dans le Haut-Rhin (France). Les installations sont composées d'une filière de traitement de type « filtres plantés de roseaux à écoulement vertical et à deux étages : FPRvv " (AGENCE DE L'EAU RHIN-MEUSE, 2010) ainsi que d'une ZRV avant le rejet dans le milieu superficiel. Le FPRvv est dimensionné sur un débit de référence de $450 \mathrm{~m}^{3}$ par jour. La STEU a été mise en service en 2009. La ZRV est de type "mare " et elle reçoit $100 \%$ des eaux la STEU (épurées, des trop-pleins et des bypass). La surface et le volume ont été estimés respectivement à $750 \mathrm{~m}^{2}$ et $425 \mathrm{~m}^{3}$. Lors de sa construction, des plantes locales et représentatives des milieux ont été plantées (AGENCE DE L'EAU RHIN-MEUSE, 2011). Le fond de la ZRV a été étanchéifié avec un voile d'argile.

\subsection{Acquisition des données météorologiques}

Une station météorologique (ADCON, Klosterneuburg, Autriche) a été implantée sur le site d'étude. Elle mesure en continu les paramètres suivants : température de l'air, hygrométrie, pression atmosphérique, rayonnement solaire, vitesse et direction du vent ainsi que la pluviométrie.

\subsection{Stratégie de prélèvement}

Tous les deux mois depuis juin 2015, des prélèvements des matrices liquides et solides ont été réalisés aussi bien sur la STEU que sur la ZRV.

\subsubsection{Matrice liquide}

Les eaux situées en entrée et sortie de la STEU (ou entrée ZRV) ainsi qu'en sortie de la ZRV ont constitué les trois 
échantillons liquides de chaque campagne de prélèvement. Les prélèvements $24 \mathrm{~h}$ ont été réalisés à l'aide de préleveurs automatiques (TELEDYNE ISCO, Lincoln, Nebraska, Éats-Unis), réfrigérés entre 0 et $1{ }^{\circ} \mathrm{C}$. L'asservissement des prélèvements au débit est rendu possible grâce aux canaux venturis (ISMA, Forbach, France) ainsi qu'aux capteurs ultrasons (IJINUS, Mel-lac, France) mesurant la hauteur d'eau et la convertissant en débit. Chacun des trois préleveurs a été équipé d'un flacon de $14 \mathrm{~L}$ permettant ainsi la collecte et le mélange des échantillons unitaires de $50 \mathrm{~mL}$.

\subsubsection{Matrices solides}

\subsubsection{Les plantes}

Les cinq plantes prélevées mensuellement étaient : l'iris, le carex, le jonc, le saule et la callitriche. Elles étaient situées dans la zone d'entrée des eaux dans la ZRV notamment sur les berges au niveau de la ligne de flottaison, exception faite de la callitriche qui est une plante subaquatique. Lorsque le développement de la plante le permettait, $150 \mathrm{~g}$ de feuilles ont été prélevés.

\subsubsection{Les boues}

Évitant la couche d'argile afin de pérenniser l'étanchéité du site, $100 \mathrm{~g}$ de boue ont été prélevés dans la couche supérieure présente au fond de l'ouvrage. Un échantillon composite fut constitué en prélevant à l'entrée, au milieu et en sortie de la ZRV.

\subsection{Extraction des médicaments}

Un volume de $100 \mathrm{~mL}$ d'échantillon de l'entrée de la STEU et $200 \mathrm{~mL}$ de l'entrée puis de la sortie de la ZRV ont été filtrés à $0,45 \mu \mathrm{m}$ en utilisant des filtres en ester de cellulose (Whatman, Maidstone, Royaume-Uni). Après acidification à $0,5 \%$ avec de l'acide acétique, les échantillons sont passés à travers des cartouches d'extraction SPE de phase inverse (C18 et HLB) (Imchem-Waters, Versailles, France) montées en série. Après que les liquides aient été filtrés, les cartouches ont été éluées avec $10 \mathrm{~mL}$ de méthanol par cartouche. Les filtrats ont été ramenés à sec pour être conservés avant analyse.

Les échantillons de plantes ont été lyophilisés et réduits en poudre alors que les échantillons de boues ont été décantés $24 \mathrm{~h}$ puis égouttés avec des filtres de type gaze. Respectivement $20 \mathrm{~g}$ de matière sèche par plante et $100 \mathrm{~g}$ de boue ont été macérés pendant $24 \mathrm{~h}$ dans $1 \mathrm{~L}$ d'acétonitrile acidifié à $0,5 \%$ (acide acétique). Les solutions ont ensuite été passées à travers des cartouches SPE de phase normale (SIO) (Imchem-Waters). Ces dernières ont été éluées avec $10 \mathrm{~mL}$ de méthanol à $20 \%$. Les filtrats ont été ramenés à sec pour être conservés avant analyse.

\subsection{Détection et quantification des médicaments}

Les analyses des médicaments ont été réalisées avec un chromatographe liquide à haute performance (UPLC) associé à un spectromètre de masse (UPLC-MS/MS, Quattro Premier XE, Waters, Saint-Quentin-en-Yvelines, France). Les méthodes analytiques ont été développées à partir des standards purs des médicaments (PETROVIC et al., 2014). La colonne chromatographique est une Acquity UPLC HSS T3 C18 colonne $(2,1 \times 100 \mathrm{~mm}, 1,8 \mu \mathrm{m}$; Waters $)$, de phase inverse. Les solvants mis en œuvre pour l'analyse étaient du méthanol pur ainsi qu'une solution de formate d'ammonium à $10 \mathrm{mM}$ acidifié à $0,1 \%$ d'acide formique. Avant l'analyse, les échantillons précédemment ramenés à sec ont été décongelés, resolubilisés avec $400 \mu \mathrm{L}$ de méthanol à $10 \%$. Cinq injections de $5 \mu \mathrm{L}$ ont été nécessaires pour quantifier les 81 molécules recherchées.

\subsection{Traitement des données}

Les concentrations de médicaments analysées dans les matrices solides s'expriment par unité de gramme de matière sèche pour les plantes et en gramme de matière égouttée pour les boues. Les capacités d'épuration des médicaments contenus dans les eaux sont exprimées en flux (concentration $\mathrm{x}$ volume journalier). Les performances épuratoires des ouvrages ainsi que la participation de la ZRV à l'épuration globale du site ont été déterminées en considérant les flux entrant et sortant des ouvrages considérés :

$$
\begin{gathered}
\text { Rendement épuratoire }_{\text {FPRvv ZRV }}=\frac{\text { Flux }_{\text {entrant }}-\text { Flux }_{\text {sortant }}}{\text { Flux }_{\text {entrant }}} \\
\text { Abattement }_{\text {ZRV }}=\frac{\text { Flux }_{\text {entrant ZRV }- \text { Flux }_{\text {sortant ZRV }}}}{\text { Flux }_{\text {entrant FPRvv }}}
\end{gathered}
$$

Des règles de calculs du rendement d'épuration (FPRvv et ZRV) ont été appliquées pour ne garder que les rendements jugés significatifs en prenant en compte les limites de quantification de chaque molécule (CHOUBERT, 2014).

\section{RÉSULTATS ET DISCUSSION}

\subsection{Caractérisations des campagnes de prélèvement}

Les campagnes de prélèvements sont synthétisées dans le tableau 1. Celles de janvier 2016 et de mai 2016 n'ont pas été réalisées à cause des ouvrages de comptage qui ont été 
Tableau 1. Caractéristiques des campagnes de prélèvement : données météorologiques et taux de charges hydrauliques (TCH) et organiques (TCO).

Table 1. Sampling campaign information: weather data, hydraulic and organic loads.

\begin{tabular}{cccccccc}
\hline Année & $\begin{array}{c}\text { Mois de } \\
\text { prélèvement }\end{array}$ & Statut & $\begin{array}{c}\text { Pluie } \\
(\mathbf{m m})\end{array}$ & $\begin{array}{c}\text { Température } \\
\left({ }^{\circ} \mathbf{C}\right)\end{array}$ & $\begin{array}{c}\text { Rayonnement solaire } \\
\left(\mathbf{W} \cdot \mathbf{m}^{-\mathbf{2}}\right)\end{array}$ & $\begin{array}{c}\text { TCH } \\
(\%)\end{array}$ & $\begin{array}{c}\text { TCO } \\
(\%)\end{array}$ \\
\hline 2015 & Juin & Réalisé & 0 & $21,9 \pm 5,9$ & $31103(2-3555)$ & 48 & 68 \\
& Septembre & Réalisé & 0 & $15,0 \pm 3,8$ & $18589,6(0-2935)$ & 36 & 66 \\
& Novembre & Réalisé & 0 & $-2,3 \pm 3,3$ & $6919,2(0-1509)$ & 42 & 26 \\
2016 & Janvier & Canaux venturis noyés & 26 & - & - & - & - \\
& Mars & Réalisé & 0 & $2,9 \pm 3,7$ & $11759,6(0-1953)$ & 51 & 29 \\
& Mai & Canaux venturis noyés & 20 & - & - & - & - \\
\hline
\end{tabular}

submergés suite aux fortes précipitations (respectivement 26 et $20 \mathrm{~mm}$ ). Les prélèvements de mars 2016 ont été faits alors que les filtres du deuxième étage de traitement du FPRvv étaient colmatés, les eaux passaient directement par les trop-pleins. Les moyennes des températures de l'air ainsi que la quantité de rayonnement solaire mettent en évidence les variations du biotope sur le fonctionnement de la ZRV. Enfin les taux de charges hydrauliques (TCH) et organiques (TCO) mettent en évidence que lors des prélèvements les installations étaient en moyenne à $44 \%$ et $47 \%$ de leurs capacités nominales.

\subsection{Bilans hydriques}

L'étude des volumes entrant et sortant de la ZRV met en évidence une capacité de l'ouvrage à réduire les flux rejetés : en moyenne une réduction de $9 \%$ du volume entrant avec un maximum en septembre 2015 (30\%) et un minimum en juin $(-19 \%)$. Ces volumes sont soient infiltrés (de façon limitée par la présence du voile d'argile), soit évaporés ou évapo-transpirés. La ZRV présente une capacité de lissage des débits sortant du FPRvv (intensité et durée) : par exemple, en novembre le pic sortant du FPRvv était de $2,9 \mathrm{~m}^{3} \cdot \mathrm{h}^{-1}$ à $2 \mathrm{~h}$ de prélèvement alors qu'en sortie de la ZRV, le pic fut de $1,6 \mathrm{~m}^{3} \cdot \mathrm{h}^{-1}$ à plus $5 \mathrm{~h} \mathrm{du}$ début du prélèvement.

\subsection{Matrice liquide : détection et quantification des médicaments}

\subsection{1 État des lieux des résidus médicamenteux dans le FPRvv}

En entrée du FPRvv, 32 à 37 médicaments ont été détectés avec les maximums pour les mois de septembre 2015 et de novembre 2015. Les concentrations en entrée étaient comprises entre le $\mathrm{mg} \cdot \mathrm{mL}^{-1}$ et le $\mathrm{ng} \cdot \mathrm{mL}^{-1}$ alors qu'en sortie elles étaient entre le $\mu \mathrm{g} \cdot \mathrm{mL}^{-1}$ et le $\mathrm{ng} \cdot \mathrm{mL}^{-1}$. L'alpha éthinylestradiol (contraceptif hormonal) est le composé le plus présent en juin $2016\left(53,99 \mu \mathrm{g} \cdot \mathrm{L}^{-1}\right)$. La théophylline (antiasthmatique et stimulant du thé) a été le composé le plus présent en septembre 2015 et mars 2016 avec respectivement 1,39 mg. $\mathrm{L}^{-1}$ et $1,12 \mathrm{mg} \cdot \mathrm{L}^{-1}$. En novembre 2016, la concentration maximum relevée fut de 2,9 $\mu \mathrm{g} \cdot \mathrm{L}^{-1}$ pour l'amitriptyline (un antidépresseur). En sortie d'ouvrage, le nombre de molécules détectées était moins important qu'en entrée avec une réduction moyenne du nombre de $19 \%$. La réduction la plus importante a eu lieu en juin (34\% des médicaments entrant) et la plus faible en mars 2016 quand le deuxième étage de traitement était colmaté (pas de réduction du nombre entrant).

\subsubsection{Présence des résidus médicamenteux dans la $Z R V$}

La réduction du nombre de molécules entre l'entrée et la sortie a été en moyenne de $7 \%$ avec un maximum de $21 \%$ en septembre 2015 et un minimum de $-3 \%$ en mars 2016. Les concentrations en entrée et en sortie étaient respectivement de l'ordre du $\mu \mathrm{g} \cdot \mathrm{mL}^{-1}$ et du $\mathrm{ng} \cdot \mathrm{mL}^{-1}$. En entrée d'ouvrage, la molécule la plus présente pour les mois de juin 2015, septembre 2015, novembre 2016 et mars 2016 fut le tramadol (antalgique) respectivement aux concentrations suivantes : $9,09 \mu \mathrm{g} \cdot \mathrm{L}^{-1}, 298,18 \mu \mathrm{g} \cdot \mathrm{L}^{-1}, 269 \mu \mathrm{g} \cdot \mathrm{L}^{-1}$ et 512,05 $\mu \mathrm{g} \cdot \mathrm{L}^{-1}$. En sortie d'ouvrage, le tramadol fut le plus présent des médicaments pour les campagnes de septembre 2015 à mars 2016 avec une concentration moyenne de 339,34 $\mu \mathrm{g} \cdot \mathrm{L}^{-1}$ (maximum de $504 \mu \mathrm{g} \cdot \mathrm{L}^{-1}$ en mars 2016). En juin 2015, l'alpha éthinylestradiol fut la concentration la plus élevée détectée à $14,9 \mu \mathrm{g} \cdot \mathrm{L}^{-1}$.

\subsection{Capacité d'épuration du FPRvv et de la ZRV}

\subsubsection{Efficacité du FPRvv}

D'après la figure 1, il y a eu une augmentation du nombre total de rendements à la période hivernale due à l'augmentation de la diversité de micropolluants dans les eaux usées. Il convient de noter la présence d'un relargage de quelques médicaments lors de chaque bilan de fonctionnement. Les mois de juin, septembre et novembre 2015 mettent en évidence la présence majoritaire de rendements épuratoires de niveau "moyen " compris entre 30 et $70 \%$. La campagne de mars 2016 met en évidence que le FPRvv présentait de nombreux relargages. 
a

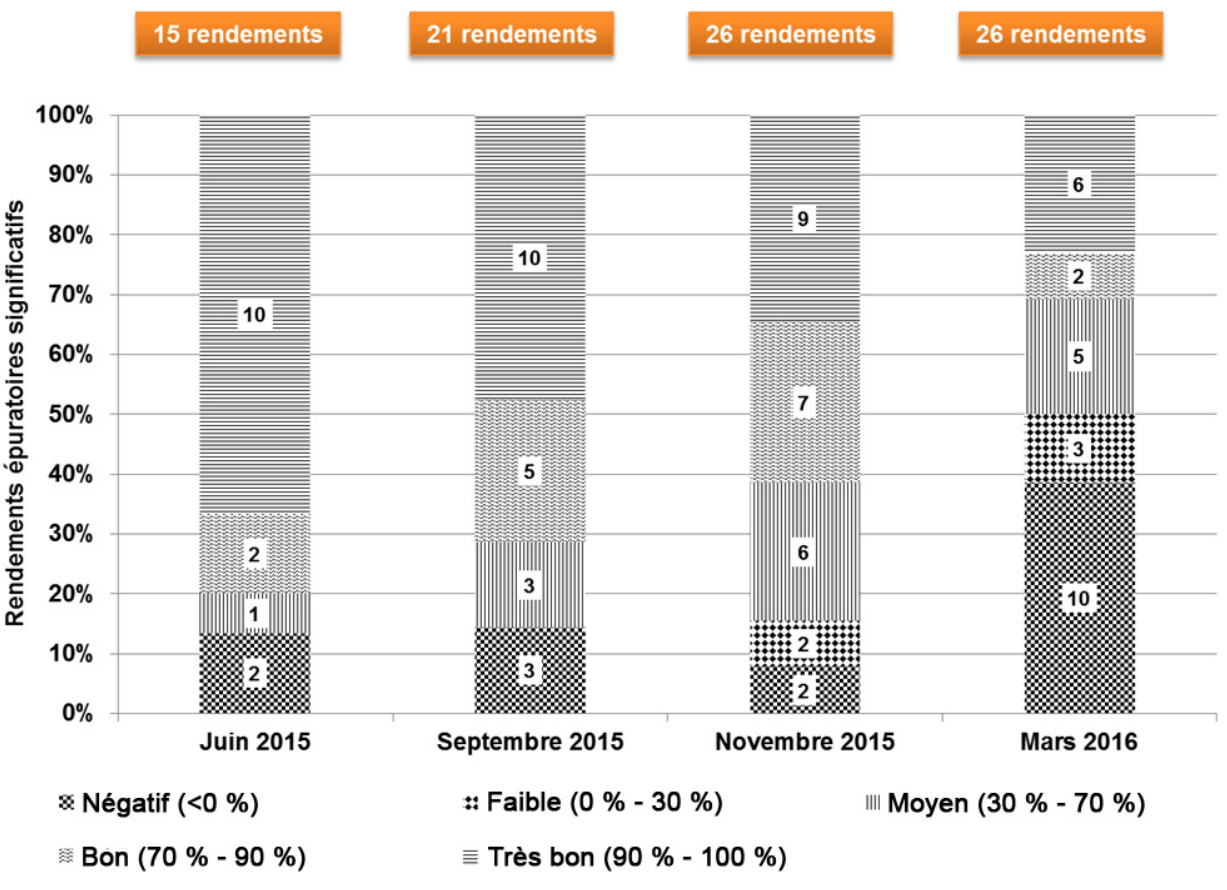

b
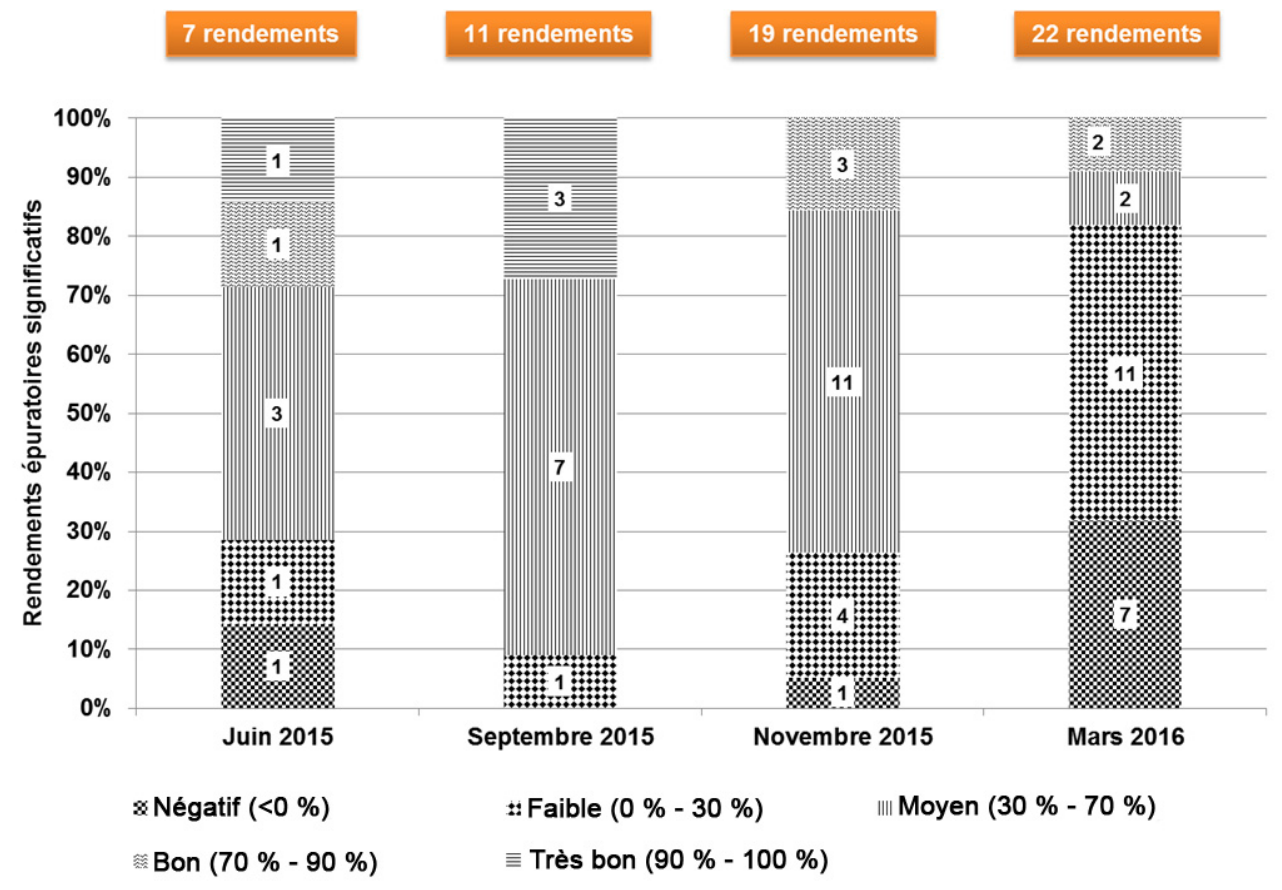

Figure 1. Rendements épuratoires significatifs regroupés par classe de performance : a) d'un filtre planté de roseaux à deux étages et écoulement vertical (FPRvv) et b) de la zone de rejet végétalisée (ZRV).

Treatment capacity efficiencies grouped by performance classes : a) for vertical flow constructed wetland, and b) for surface flow treatment wetland.

\subsubsection{La complémentarité de la ZRV}

D'après la figure 1, il y a : une augmentation de la diversité de substances médicamenteuses abattue vers fin de la période hivernale (de 7 à 22), quelques relargages de médicaments par campagne et trois campagnes avec une majorité de rendements de niveau "moyen ». La campagne de mars 2016 met en évidence de faibles performances avec des rendements épuratoires de niveau "faible " et " nul " majoritairement présents. Lors de cette campagne, il n'y a pas eu de réduction des volumes hydrauliques. 


\subsection{Capacités d'épuration complémentaires de la ZRV au FPRvv}

Dans la figure 2, les rendements épuratoires de la ZRV ont été exprimés par rapport aux flux de pollution entrants dans le FPRvv. Ces derniers ont été regroupés par classe de performances pour chaque campagne de prélèvement. La participation de la ZRV à l'épuration globale de la STEUZRV était majoritairement comprise entre 0 et $30 \%$ pour la campagne de juin 2015 et entre 30 et $70 \%$ pour les campagnes de septembre 2015 et de novembre 2015. Un relargage a eu lieu aux campagnes de juin 2015 (-49,38 \% de carbamazépine [antiépileptique/normothymique/antalgique]) et de novembre 2015 (-0,58 \% d'ofloxacin [antibiotique]). En mars 2016, malgré le dysfonctionnement du FPRvv, la ZRV a tout de même permis de retenir et/ou dégrader des résidus médicamenteux entre 0 et $30 \%$ avant rejet des eaux dans le milieu superficiel.

\subsection{Dissipation des médicaments de la matrice liquide vers les matrices solides}

La diversité des résidus médicamenteux dans les plantes est comprise entre 10 et 22 alors que dans le sol elle était comprise entre 8 et 19. A chaque campagne l'iris et le jonc étaient les plantes présentant le plus grand nombre de médicaments (respectivement 22 et 21 molécules en moyenne). Les concentrations de médicaments détectées étaient comprises entre le ng. $\mathrm{g}^{-1}$ et le $\mathrm{pg} \cdot \mathrm{g}^{-1}$ avec une redondance du tramadol et de l'ibuprofène (antalgique/anti-inflammatoire). Les plantes et le sol présentaient une diversité de médicaments supérieure à celle présente dans les eaux mettant en évidence une capacité d'accumulation de ces micropolluants au cours du temps.

\section{CONCLUSION}

Les STEU des petites communes sont soumises à la pollution de résidus pharmaceutiques à des concentrations comprises entre le $\mu \mathrm{g} \cdot \mathrm{mL}^{-1}$ et le ng. $\mathrm{mL}^{-1}$. Les FPRvv retiennent et/ou dégradent une partie de ses micropolluants mais à des proportions propre à chaque molécule. De plus, les flux entrants dans la ZRV sont diminués par évaporation, l'évapotranspiration et l'infiltration. Un des processus de la diminution des concentrations est le stockage de ces composés dans les boues et les plantes présentes dans le milieu. Toutefois, ce transfert semble négligeable au vu des flux entrants dans la ZRV qui sont de l'ordre du gramme par jour alors que les matrices solides ont des concentrations de l'ordre du pg.g ${ }^{-1}$ de matière sèche.

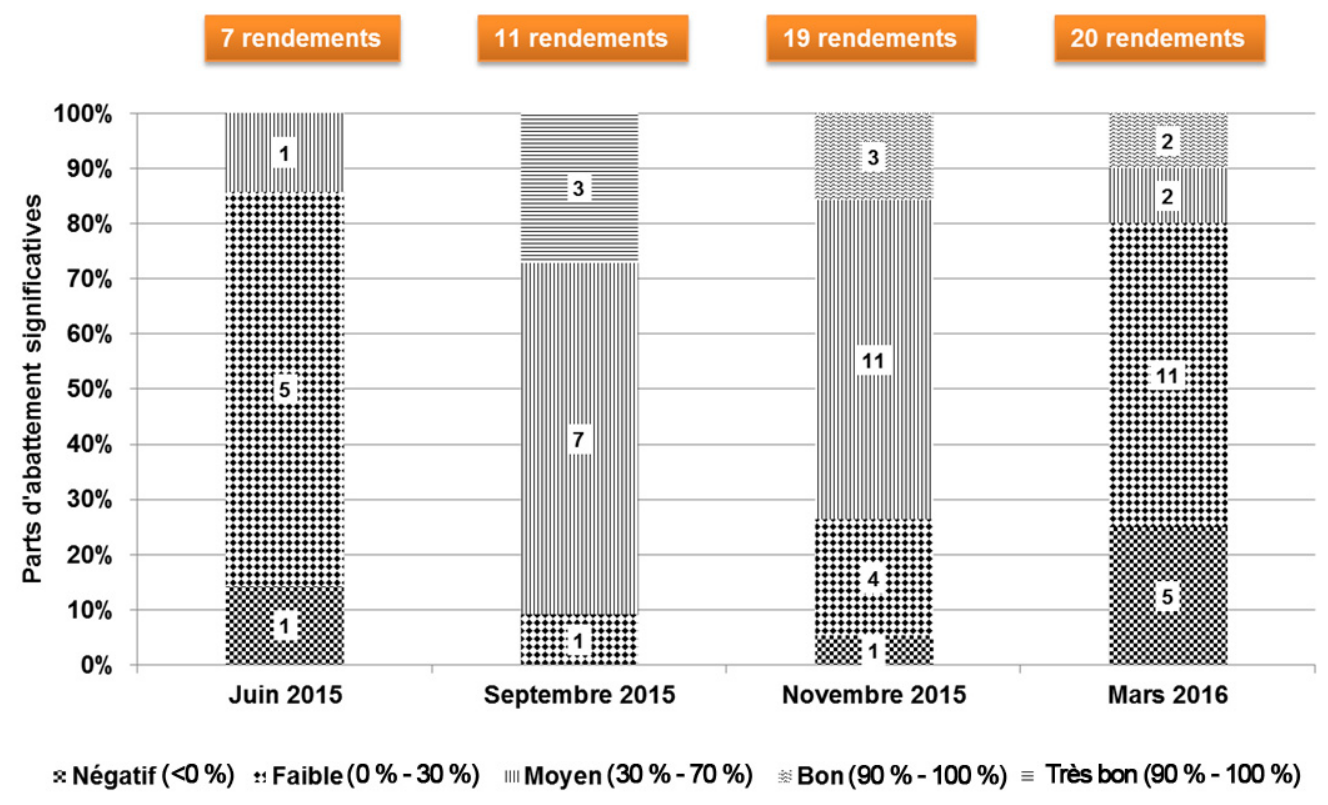

Figure 2. Parts d'abattement complémentaires de la zone de rejet végétalisée (ZRV) à l'épuration d'un filtre planté de roseaux à deux étages et écoulement vertical (FPRvv). Surface flow treatment wetland additional contribution to vertical flow constructed wetland efficiency capacity. 


\section{RÉFÉRENCES BIBLIOGRAPHIQUES}

AGENCE DE L'EAU RHIN-MEUSE (2010). Guide méthodologique pour l'assainissement des agglomérations de moins de 2000 équivalents-habitants. Agence de l'eau RhinMeuse, France, 46 p.

AGENCE DE L'EAU RHIN-MEUSE (2011). Zone de rejet végétalisée: Aménagement des milieux naturels en aval de station d'épuration. Agence de l'eau Rhin-Meuse, France, $10 \mathrm{p}$.

BOUTIN, C. et S. PROST-BOUCLE (2012). Note de présentation : Les zones de rejet végétalisées. Sciences Eaux \& Territoires, 9, 36-43.

CHOUBERT, J.M. (2014). Rendements d'élimination d'un fossé et de filtres garnis de matériaux adsorbants. Colloque final du projet de recherche ARMISTIQ, 6 février, Villeurbanne, France, 17 p. https://armistiq.irstea.fr/wp-content/ uploads/2014/02/05-ARMISTIQ_Tertiaires-extensifsVsite.pdf

LARSSON, D.G.J., M. ADOLFSSON-ERICI, J. PARKKONEN, M. PETTERSSON, A.H. BERG, P.E. OLSSON et L. FÖRLIN (1999). Ethinyloestradiol - an undesired fish contraceptive? Aquat. Toxicol., 45 (2-3), 9197.
LI, W.C. (2014). Occurrence, sources, and fate of pharmaceuticals in aquatic environment and soil. Environ. Pollut., 187, 193-201.

PETROVIC, M., B. ŠKRBIC, J.ŽIVANCEV, L. FERRANDOCLIMENT et D. BARCELO (2014). Determination of 81 pharmaceutical drugs by high performance liquid chromatography coupled to mass spectrometry with hybrid triple quadrupole-linear ion trap in different types of water in Serbia. Sci. Total Environ., 468-469, 415-28.

PURDOM, C.E., P.A. HARDIMAN, V.V.J. BYE, N.C. ENO, C.R. TYLER et J.P. SUMPTER (1994). Estrogenic effects of effluents from sewage treatment works. Chem. Ecol., 8 (4), 275-285.

SUMPTER, J.P., et S. JOBLING (1995). Vitellogenesis as a biomarker for estrogenic contamination of the aquatic environment. Environ. Health Persp., 103 (7), 173-178.

ZHANG, D., R.M. GERSBERG, W.J. NG et S.K. TAN (2014). Removal of pharmaceuticals and personal care products in aquatic plant-based systems: A review. Environ. Pollut., 184, 620-639. 